\title{
Changes in Mid-regional pro-atrial natriuretic peptide (MR-proANP) during thirsting separate patients with diabetes insipidus from those with primary polydipsia
}

Sandrine Urwyler ${ }^{1 *}$, Katharina Timper ${ }^{1+2^{*}}$, Wiebke Fenske ${ }^{3}$, Felix Kühn ${ }^{4}$, Nica Frech ${ }^{1}$, Birsen Arici ${ }^{5}$, Jonas Rutishauser ${ }^{6}$, Peter Kopp ${ }^{7}$, Bruno Allolio ${ }^{8}$, Christoph Stettler ${ }^{4}$, Beat Müller ${ }^{9}$, Mira Katan $^{10}$ and Mirjam Christ-Crain ${ }^{1}$

${ }^{1}$ Clinic of Endocrinology, Diabetes and Metabolism, Department of Clinical Research, University Hospital Basel, Switzerland, ${ }^{2}$ Max-Planck-Institute for Metabolism Research, Cologne, Germany, ${ }^{3}$ ntegrated Research and Treatment Center for Adiposity Diseases, Leipzig University Medical Center, Germany, ${ }^{4}$ Division of Endocrinology, Diabetes and Clinical Nutrition, University Hospital Bern - Inselspital, Switzerland, ${ }^{5}$ Department of Internal Medicine, Spital Rheinfelden, Switzerland, 6University Clinic of Internal Medicine, Kantonsspital Baselland, Bruderholz, Switzerland, ${ }^{7}$ Division of Endocrinology, Metabolism and Molecular Medicine and Center for Genetic Medicine, Northwestern University, Chicago, USA, ${ }^{8}$ Department of Internal Medicine I, Endocrine and Diabetes Unit, University Hospital Würzburg, Germany,

${ }^{9}$ Division of Endocrinology, Diabetology and Metabolism, Medical University Clinic, Kantonsspital Aarau, Switzerland, ${ }^{10}$ Department of Neurology, University Hospital Zurich, Switzerland, *equally contributing

\section{Background \& Aim}

\section{Background:}

The water deprivation test as the accepted gold standard in the differential diagnosis of polyuria polydipsia syndrome can be associated with a decrease in extracellular fluid volume in patients with diabetes insipidus (DI).

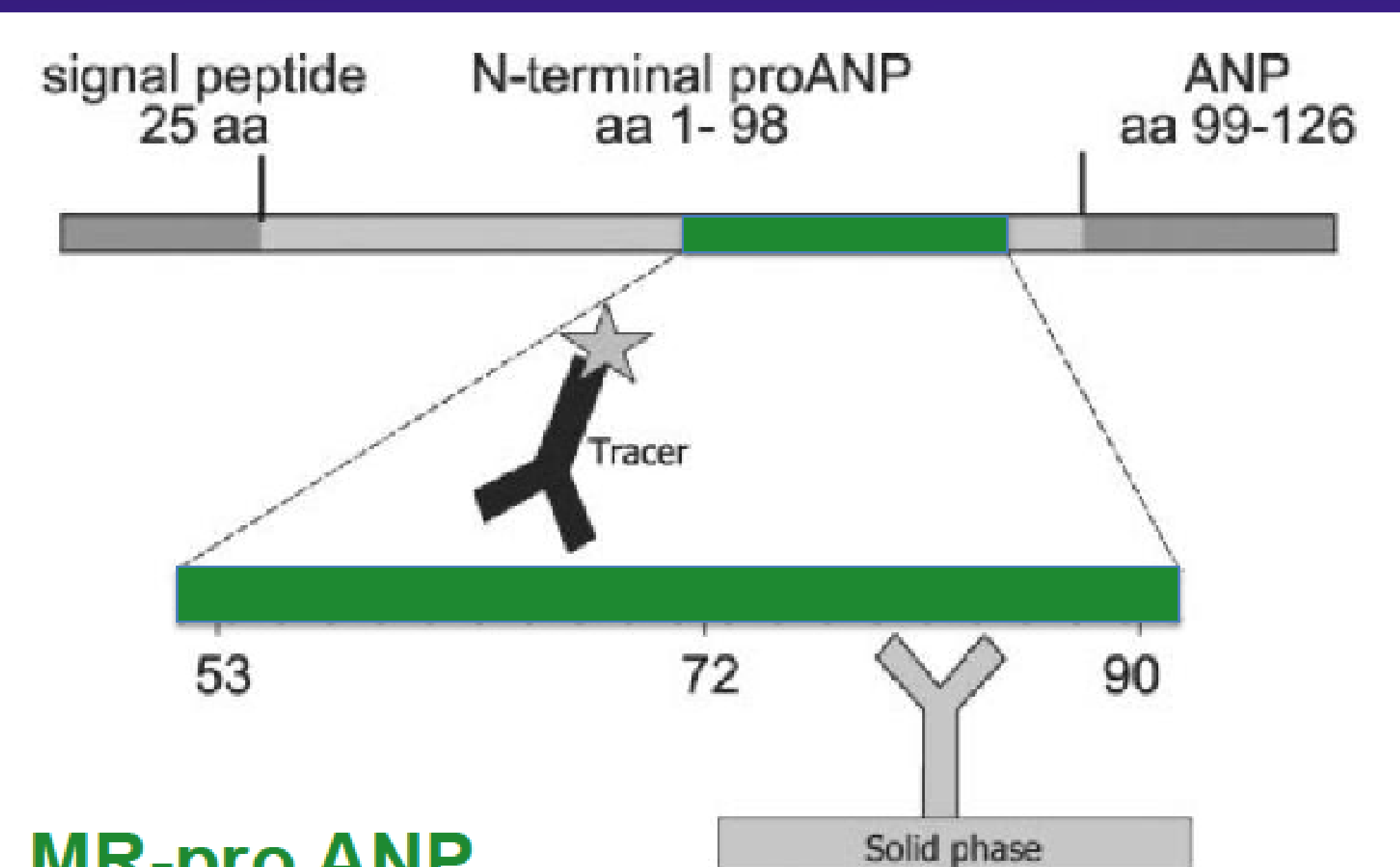

MR-pro ANP
Aim:

To evaluate mid-regional pro-atrial natriuretic peptide (MR-proANP) as marker of extracellular body volume in patients with diabetes insipidus compared to primary polydipsia (PP) at baseline and after water deprivation.

\section{Methods}

\section{Patients included:}

Age $>18$ years

History of polyuria ( $>40 \mathrm{~mL} / \mathrm{kg} / 24 \mathrm{~h})$ and polydipsia

Methods:

- Participants underwent a standardized combined water deprivation and $3 \%$ saline infusion test that was terminated when serum sodium levels exceeded $147 \mathrm{mmol} / \mathrm{L}$.

MR-proANP levels were determined at baseline and when serum sodium exceeded $147 \mathrm{mmol} / \mathrm{L}$.

\section{Results}

Table 1: Baseline Characteristics

\begin{tabular}{|c|c|c|c|c|}
\hline & $\begin{array}{l}\text { All Patients } \\
\quad(n=48)\end{array}$ & $\begin{array}{l}\text { Diabetes } \\
\text { insipidus } \\
(n=31)\end{array}$ & $\begin{array}{l}\text { Primary Polydipsia } \\
\qquad(n=17)\end{array}$ & p-value \\
\hline Age (years), median (IQR) & $45(33 ; 57)$ & $48(39 ; 59)$ & $36(27 ; 46)$ & 0.06 \\
\hline BMI (kg/m2) & $25.2(22.0 ; 30.5)$ & $24.8(22.2 ; 31.8)$ & $25.5(19.6 ; 27.5)$ & 0.22 \\
\hline Gender, male/female, (female \%) & $27(56.3 \%)$ & $15(48.3 \%)$ & $12(70.6 \%)$ & 0.22 \\
\hline Current smoker (yes \%) & $13(27.1 \%)$ & $8(25.8 \%)$ & $5(29.4 \%)$ & 1.0 \\
\hline History of a brain tumor (yes \%) & $10(20.8 \%)$ & $10(32.3 \%)$ & $0(0 \%)$ & 1.0 \\
\hline $\begin{array}{l}\text { History of transsphenoidal surgery } \\
(\text { yes \%) }\end{array}$ & $9(18.8 \%)$ & $9(29 \%)$ & $0(0 \%)$ & 0.02 \\
\hline Blood pressure systolic (mmHg) & $125(115 ; 135)$ & $127(120 ; 139)$ & $113(106 ; 130)$ & 0.04 \\
\hline Blood pressure diastolic $(\mathrm{mmHg})$ & $80(74 ; 85)$ & $80(75 ; 87)$ & $75(70 ; 84)$ & 0.13 \\
\hline Heart rate $(/ \mathrm{min})$ & $71(65 ; 80)$ & $70(64 ; 77)$ & $79(66 ; 84)$ & 0.11 \\
\hline Body temperature ( C) & $36.8(36.4 ; 37.4)$ & $36.6(36.4 ; 37.0)$ & $37.3(36.8 ; 37.7)$ & 0.03 \\
\hline Fluid intake, L/24h & $6(4 ; 8)$ & $6(4.5 ; 8.5)$ & $4.5(4 ; 7)$ & 0.27 \\
\hline $\begin{array}{c}\text { Clinical volume status: } \\
\text { Hypo-/eu-/hypervolaemic } \\
\text { (euvolaemic \%) }\end{array}$ & $\begin{array}{l}5 / 34 / 0 \\
(87.2 \%)\end{array}$ & $\begin{array}{l}3 / 25 / 0 \\
(89.3 \%)\end{array}$ & $\begin{array}{l}2 / 10 / 0 \\
(83.3 \%)\end{array}$ & 0.63 \\
\hline Hemoglobin $(g / l)$ & $139(133 ; 147)$ & $138(134 ; 148)$ & $142(134 ; 144)$ & 0.89 \\
\hline Hematocrit (I/I) & $0.41(0.38 ; 0.43)$ & $0.41(0.38 ; 0.43)$ & $0.41(0.39 ; 043)$ & 1.0 \\
\hline Creatinine (umol/l) & $73(64 ; 85)$ & $80(65 ; 88)$ & $66(62 ; 73)$ & 0.01 \\
\hline Albumine (g/l) & $40(37 ; 43)$ & $40(37 ; 43)$ & $41(37 ; 43)$ & 0.85 \\
\hline Diuretics (\%) & $1(2.1 \%)$ & $0(0 \%)$ & $1(5.9 \%)$ & 0.35 \\
\hline Lithium (\%) & $5(10.4 \%)$ & $4(12.9 \%)$ & $1(5.9 \%)$ & 0.64 \\
\hline Antiepileptics (\%) & $4(8.3 \%)$ & $2(6.5 \%)$ & $2(11.8 \%)$ & 0.61 \\
\hline Desmopressin (\%) & $7(14.6 \%)$ & $7(22.6 \%)$ & $0(0 \%)$ & 0.04 \\
\hline
\end{tabular}

MR-proANP in Diabetes insipidus and Primary Polydipsia

Delta-MR-proANP in Diabetes insipidus and Primary Polydipsia
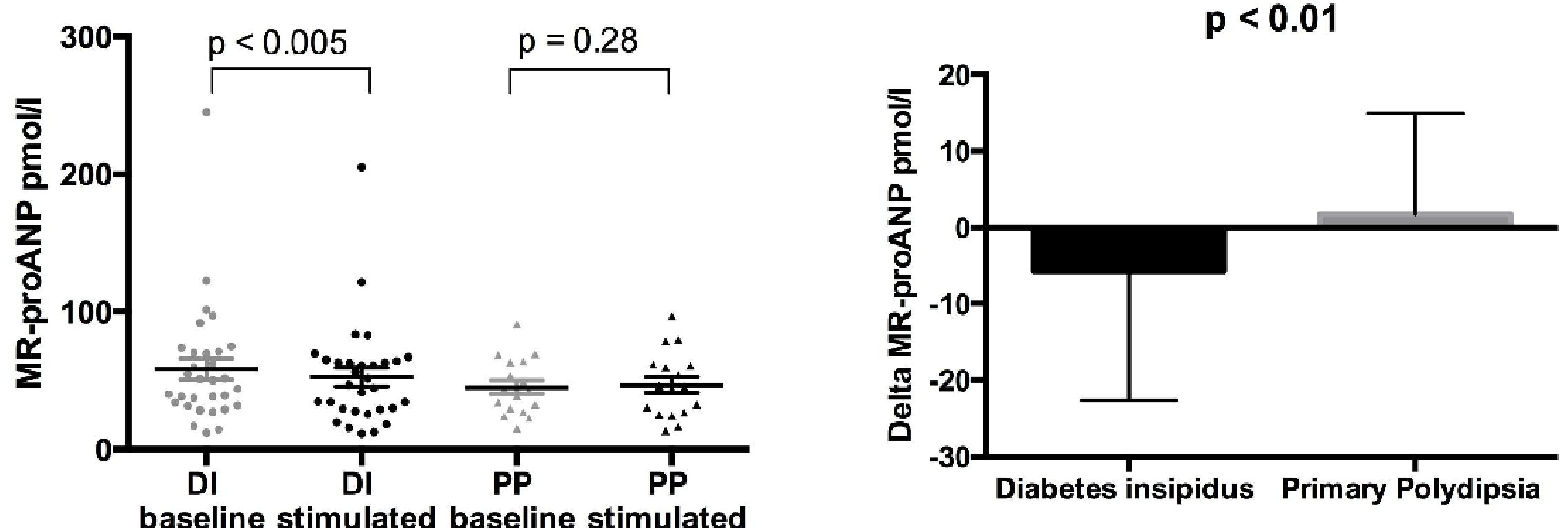

ROC curve of Delta-MR-proANP to differentiate Diabetes insipidus from Primary Polydipsia

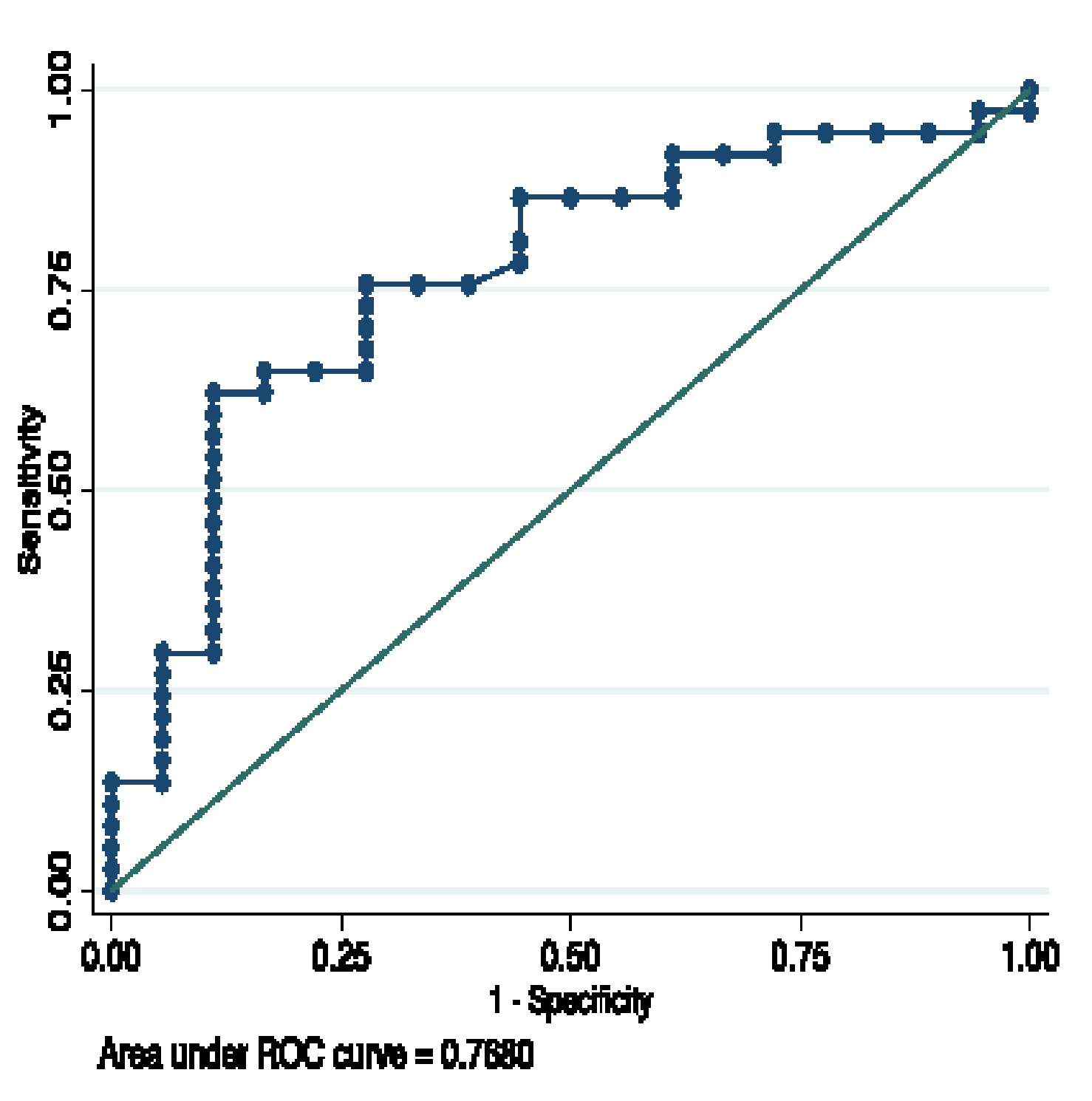

\section{Summary \& Conclusion}

MR-proANP levels decrease upon a water deprivation test in patients with DI, without a change in PP. MR-proANP levels mirror the subtle volume loss in patients with DI undergoing a water deprivation test.

Delta-MR-proANP was able to predict diagnosis of $\mathrm{DI}$ in a water deprivation test by reflecting change in volume status.

Therefore, Delta-MR-proANP, in combination with other markers (e.g. copeptin, vasopressin) should be further evaluated as promising laboratory parameter in the diagnosis and differential diagnosis of polyuria polydipsia syndrome. 